\title{
Adversidad psicosocial, salud mental y suicidio en adolescentes: ¿estamos haciendo lo suficiente para atender a esta población?
}

\author{
Lino Palacios Cruz ${ }^{1}$
}

Editorial

"El mundo es un buen lugar por el que vale la pena luchar..." Ernest Hemingway, 1899-1961

Cada 10 de septiembre, desde el 2003, la Asociación Internacional para la Prevención del Suicidio https:/ / www.iasp. info/es/index.php y la Organización Mundial de la Salud (OMS) promueven el Día Mundial para la Prevención del Suicidio, con el objetivo de concientizar a la población en general que el suicidio puede presentarse desde edad temprana y es una condición que puede prevenirse. ${ }^{1}$ El suicidio es una de las principales causas de muerte en el mundo, aunque su etiología aún no es del todo bien entendida.

En este número de SALUD MENTAL se presentan interesantes trabajos relacionados con tópicos como la evaluación de la efectividad de las intervenciones psicosociales en ámbitos como el estrés o la esquizofrenia, el estudio de aspectos psicométricos relacionados con la evaluación de la percepción y la memoria musical, con la determinación de variables relacionadas a acceso a servicios de salud mental, y la evaluación neuropsicológica o de la salud mental en grupos en alto riesgo. Sin embargo, un tema que en especial atrae mi atención es aquel que tiene que ver con el estudio de la conducta suicida en niños y adolescentes $y$, finalmente, su relación con la salud mental en dicha población.

La conducta suicida, incluida las autolesiones sin un claro fin suicida, es un problema de salud pública, y por ello, en la mayor parte del mundo, la tasa de suicidios es considerada como un indicador de la salud mental de la población. ${ }^{2}$ En nuestro país, desde 1970 a la fecha, ha existido un aparente incremento, al menos en el reporte, de casos de suicidio, de un $275 \%$ hasta el 2007. Según Borges et al., en un estudio previamente publicado, el suicidio en nuestro país se había incrementado en el lapso de 1970 a 2007, principalmente en el grupo de 15 a 29 años. ${ }^{3}$ Los autores del presente trabajo de revisión que abarca de 1998 al 2011, analizan las bases de defunciones del Sistema Nacional de Información en Salud y las proyecciones de población del Consejo Nacional de Población. En la población de cinco a 19 años el sui- cidio representó el 7\% de las muertes por lesiones de causa externa, siendo la razón hombre/mujer de 2.2:1, sin embargo las mujeres presentaron el mayor porcentaje de cambio con el $6 \%$.

La evidencia científica consistentemente ha mostrado que 1) los factores medioambientales o experienciales también contribuyen en el inicio y la persistencia de las conductas suicidas, 2) los factores medioambientales, llámese adversidad psicosocial, tienen una asociación más fuerte que los factores genéticos ya estudiados con desenlaces en salud mental negativos, 3) la presencia de adversidades en la infancia se relaciona con la conducta suicida en la adultez. Un trabajo reciente, basado en las encuestas en población general auspiciadas por la OMS, mostró que el riesgo de intento o ideación suicida se incrementó hasta seis veces más, conforme aumentó el número de adversidades psicosociales, especialmente aquellas consideradas como intrusivas y agresivas. $^{4}$

Los autores de la revisión que se presenta en este número de la revista señalan dos aspectos importantes: a) el incremento de casos de suicidio en adolescentes, además de que los métodos utilizados parecen ser más violentos y, b) el sub registro que persiste en nuestro país. Además nos invitan a pensar sobre lo importante que es la incorporación de una evaluación de la salud mental, desde muy temprana edad, en el cuidado de la salud integral de todo individuo. Dicha vigilancia de niños y adolescentes necesariamente tendría que incluir el primer nivel de atención en salud, evaluando especialmente a aquellos individuos que forman parte de grupos de alto riesgo para problemas en salud mental, como podrían ser los hijos de padres con psicopatología. La literatura científica muestra que la conducta suicida tiende a ocurrir en las familias, es decir pareciera que existe una transmisión intergeneracional del riesgo suicida; además se ha mostrado, tanto en estudios nacionales como internacio-

Subdirección de Investigaciones Clínicas, Instituto Nacional de Psiquiatría Ramón de la Fuente Muñiz. 
nales, que la psicopatología parental es un factor de riesgo para el desarrollo de conducta suicida en los descendientes de pacientes con trastornos mentales; ${ }^{5,6}$ principalmente los trastornos mentales en los padres, los cuales se caracterizan por agresión impulsiva y ansiedad/agitación, éstos son los que con mayor consistencia se han relacionado con la predicción de la conducta suicida a lo largo de la vida en sus hijos. ${ }^{7}$ La investigación en esta área ha ido más allá, señalando incluso que la conducta suicida materna es un factor de riesgo más potente que aquella encontrada en el padre, para encontrar dicha conducta en sus hijos, además de que los niños son aún más vulnerables que los adolescentes. ${ }^{8}$

En años recientes, dentro del estudio de la conducta suicida, se ha incorporado la evaluación de las autolesiones sin un fin suicida (NSSI, por sus siglas en inglés), es decir aquellas lesiones que obedecen al acto de auto-infligirse deliberadamente dolor y daño en los tejidos de un individuo en ausencia de ideación suicida, ha cobrado mayor interés debido a que se presenta frecuentemente en adolescentes y se asocia con una mayor severidad de la psicopatología. ${ }^{9} \mathrm{La}$ evidencia científica ha mostrado que entre los adolescentes que presentan algún tipo de NSSI existe un mayor riesgo tanto de intentar como de consumar el suicidio. ${ }^{10} \mathrm{Al}$ respecto, un estudio publicado en el 2006 mostró que 70 \% de los adolescentes que presentan NSSI tenía al menos el antecedente de un intento suicida previo; el 55\% de la misma muestra manifestaba el antecedente de múltiples intentos suicidas. ${ }^{11}$ Recientemente se ha mostrado también que los intentos suicidas dentro del grupo de hijos de padres con psicopatología son predichos con mayor fuerza cuando los adolescentes presentan NSSI en comparación con un intento suicida previo. ${ }^{9}$ Los adolescentes con NSSI con frecuencia presentan trastornos afectivos, ansiosos, alimentarios, trastornos externalizados e incluso trastornos por uso de sustancias. ${ }^{10}$

La identificación y el desarrollo de instrumentos clinimétricos para la población adolescente que podría encontrarse especialmente en un riesgo elevado para presentar conducta suicida, es un tema que debe ser abordado en futuras investigaciones. Algunos esfuerzos se han hecho en otros países, principalmente desarrollados, para tratar de identificar, en las salas de urgencia de los hospitales generales, a estos adolescentes en riesgo; encontrando que especialmente aquellos jóvenes que presentan el antecedente de ideación o intento suicida, además de depresión y un tras- torno por uso de alcohol, tienen una mayor probabilidad de verse involucrados en un nuevo episodio dentro de la conducta suicida. ${ }^{12}$

Finalmente, los hallazgos que son mostrados en este número de SALUD MENTAL refuerzan la importancia de considerar, a la luz del incremento en los niveles de violencia y adversidad psicosocial en nuestro país, el cuidado y vigilancia de la salud mental en niños y adolescentes y, en especial, el estudio del fenómeno de la conducta suicida desde etapas tan tempranas como la infancia.

\section{REFERENCIAS}

1. Wikipedia Cd. Día Mundial para la Prevención del Suicidio: Wikipedia, La enciclopedia libre.; [updated 12 de septiembre del 2015, 00:26 UTC. Available from: https://es.wikipedia.org/w/index.php?title=D\%C3\%ADa_Mundial_para_la_Prevenci\%C3\%B3n_del_Suicidio\&oldid=85063058.

2. INEGI. Estadísticas a propósito del... Día Mundial para la prevención del suicidio (10 de septiembre)2015. Available from: http://www.inegi. org.mx/saladeprensa/aproposito/2015/suicidio0.pdf

3. Borges G, Orozco R, Benjet C, Medina-Mora ME. [Suicide and suicidal behaviors in Mexico: Retrospective and current status]. Salud Publica Mex 2010;52(4):292-304.

4. Bruffaerts R, Demyttenaere K, Borges G, Haro JM et al. Childhood adversities as risk factors for onset and persistence of suicidal behaviour. Br J Psychiatry 2010;197(1):20-7.

5. Borges G, Borges R, Medina-Mora ME, Benjet C et al. Parental psychopathology and offspring suicidality in Mexico. Arch Suicide Res 2013;17(2):123-135.

6. Gureje O, Oladeji B, Hwang I, Chiu WT et al. Parental psychopathology and the risk of suicidal behavior in their offspring: results from the World Mental Health surveys. Mol Psychiatry 2011;16(12):1221-1233.

7. Santana GL, Coelho BM, Borges G, Viana MC et al. The influence of parental psychopathology on offspring suicidal behavior across the lifespan. PLoS One 2015;10(7):e0134970.

8. Geulayov G, Gunnell D, Holmen TL, Metcalfe C. The association of parental fatal and non-fatal suicidal behaviour with offspring suicidal behaviour and depression: a systematic review and meta-analysis. Psychol Med 2012;42(8):1567-1580.

9. Cox LJ, Stanley BH, Melhem NM, Oquendo MA et al. A longitudinal study of nonsuicidal self-injury in offspring at high risk for mood disorder. J Clin Psychiatry 2012;73(6):821-828.

10. Peterson J, Freedenthal S, Sheldon C, Andersen R. Nonsuicidal self injury in adolescents. Psychiatry (Edgmont) 2008;5(11):20-26.

11. Nock MK, Joiner TE, Gordon KH, Lloyd-Richardson E et al. Non-suicidal self-injury among adolescents: diagnostic correlates and relation to suicide attempts. Psychiatry Res 2006;144(1):65-72.

12. King CA, Berona J, Czyz E, Horwitz AG et al. Identifying adolescents at highly elevated risk for suicidal behavior in the emergency department. J Child Adolesc Psychopharmacol 2015;25(2):100-108. 